\title{
UN GLYPTODONTIDAE DE GRAN TAMAÑO EN EL HOLOCENO TEMPRANO DE LA REGIÓN PAMPEANA, ARGENTINA
}

\author{
LEOPOLDO HÉCTOR SOIBELZON, MARTÍN ZAMORANO, GUSTAVO JUAN SCILLATO-YANÉ \\ División Paleontología de Vertebrados, Museo de La Plata, Paseo del Bosque s/nº, 1900, La Plata, Argentina. \\ lsoibelzon@fcnym.unlp.edu.ar, marzamorano@fcnym.unlp.edu.ar, scillato@fcnym.unlp.edu.ar \\ DAVID PIAZZA \\ Repositorio Paleontológico “Carlos Rusconi”, Secretaría de Cultura y Educación, La Matanza, Argentina. \\ dspiazza@hotmail.com

\section{SERGIO RODRÍGUEZ} \\ Facultad de Ciencias Naturales y Museo, Universidad Nacional de La Plata, La Plata, Argentina. \\ sergio_rodriguez133@hotmail.com
}

\section{ESTEBAN SOIBELZON, EDUARDO PEDRO TONNI}

División Paleontología de Vertebrados, Museo de La Plata, Paseo del Bosque s/nº 1900, La Plata, Argentina. esoibelzon@fcnym.unlp.edu.ar,eptonni@fcnym.unlp.edu.ar

\section{JORGE SAN CRISTÓBAL}

Facultad de Ciencias Naturales y Museo, Universidad Nacional de La Plata, La Plata, Argentina.jorgeosancristobal@gmail.com

\author{
ELISA BEILINSON
}

Centro de Investigaciones Geológicas, CONICET, calle 1,644, La Plata, Argentina. beilinson@cig.museo.unlp.edu.ar

\begin{abstract}
A LARGE GLYPTODONTIDAE IN THE EARLY HOLOCENE OF THE PAMPEAN REGION, ARGENTINA. We present here the record of a very large glyptodont (1,900 to 2,370 kg); it is a right humerous distal epiphysis (RPCR 1021) coming from the early Holocene $\left(8,480 \pm 130\right.$ years ${ }^{14} \mathrm{C}$ cal. BP) of the Pampean Region (Los Pozos, Marcos Paz, Buenos Aires Province, Argentina) and we assign it to Doedicurus Burmeister cf. D. Clavicaudatus (Owen). The youngest record for a glyptodont $\left(7,839 \pm 102\right.$ years ${ }^{14} \mathrm{C}$ cal. BP) corresponds to Doedicurus clavicaudatus. Finally we discuss the possible explanations of the great size attained by glyptodonts at the end of the Lujanian age (late Pleistocene-early Holocene).
\end{abstract}

Key words: South America, Quaternary, Doedicurus, Holocene.

RESUMO - É dado a conhecer um exemplar de grande tamanho de gliptodonte (massa corporal estimada entre 1900 a $2370 \mathrm{~kg}$ ) atribuído a Doedicurus cf. D. clavicaudatus (Owen). Trata-se de uma porção distal de úmero direito (RPCR 1021), procedente do Holoceno inicial $\left(8.480 \pm 130\right.$ anos ${ }^{14} \mathrm{C}$ cal. AP) da Região Pampeana, Los Pozos, partido de Marcos Paz, Província de Buenos Aires, Argentina. O registro mais moderno para os gliptodontes $\left(7.839 \pm 102\right.$ anos ${ }^{14} \mathrm{C}$ AP) corresponde a esta mesma espécie. Finalmente, são discutidas as explicações acerca do aumento de tamanho dos gliptodontes para o final do Lujanense (Pleistoceno final-Holoceno inicial).

Palavras-chave: América do Sul, Quaternário, Doedicurus, Holoceno.

\section{INTRODUCCIÓN}

Los gliptodontes (Glyptodontidae, Xenarthra) fueron elementos muy frecuentes entre los megaherbívoros del Cenozoico de América del Sur (Paula Couto, 1979; Zurita et al., 2010). Durante el Plioceno tardío y Pleistoceno tuvieron una amplísima distribución latitudinal en América del Sur; se los encuentra desde la Argentina hasta Venezuela (Carlini \&
Zurita, 2010). Entre los gliptodontes del Cenozoico Superior, sólo Doedicurus Burmeister, 1874, Neosclerocalyptus Paula Couto, 1954 y Glyptodon Owen, 1839 se registran durante el Holoceno (Politis \& Gutiérrez, 1998; Gutiérrez et al., 2010; Cione et al., 2009). Todos los gliptodontes, junto a otros 43 géneros de megamamíferos y grandes mamíferos, se extinguieron en América del Sur entre fines del Pleistoceno y comienzos del Holoceno (Cione et al., 2009). Recientemente 
Coltorti et al. (2012) publicaron una serie de dataciones asociadas a fauna extinta, si bien utilizan taxones que carecen actualmente de validez (e.g. Clamydotherium Lund, 1839), además de utilizar el término "megafauna" (mamíferos de más de una tonelada) para referirse a Equus Linnaeus, 1758 y ser sus conclusiones discutibles, los fechados radiocarbónicos que presentan indican la supervivencia de algunos taxones extintos durante el Holoceno.

Doedicurus clavicaudatus (Owen, 1847) es el gliptodonte con registro más moderno, ya que varias dataciones radiocarbónicas lo ubican en torno a 8.000-7.000 años ${ }^{14} \mathrm{C} \mathrm{AP}$, habiéndose aceptado como más probable una de $7.010 \pm 100$ años ${ }^{14} \mathrm{C}$ AP (7.839 \pm 102 años cal. AP; véase Politis \& Gutiérrez, 1998). Sin embargo, Rosello et al. (1999) refieren una edad de 4.300 \pm 90 años ${ }^{14} \mathrm{C}$ AP $(4.866 \pm 107$ años cal. AP) para restos de Glyptodon clavipes Owen, 1839 provenientes de la Provincia de Buenos Aires. La validez de esta fecha fue cuestionada por Cione et al. (2001), basándose fundamentalmente en la calidad de la muestra datada.

Durante el Pleistoceno algunos gliptodontes poseían masas corporales de al menos $250 \mathrm{~kg}$ (e.g. Neosclerocalyptus), mientras que otros sobrepasaban los $1000 \mathrm{~kg}$ (e.g. Panochthus intermedius Lydekker, 1895; Glyptodon elongatus Burmeister, 1866; Doedicurus clavicaudatus; véase Fariña, 1995; Fariña et al. 1998; Fariña \& Vizcaíno, 1999). Todos los gliptodontes parecen haber sido formas pastadoras especializadas (Vizcaíno \& Bargo, 1998; Vizcaíno et al., 2004).

En este trabajo se da a conocer una epífisis distal de húmero asignada a Doedicurus cf. D. clavicaudatus (RPCR 1021). El material se caracteriza por el tamaño, que excede al de los numerosos ejemplares de la especie con que se comparó. Valvas del gasterópodo Biomphalaria Preston, 1910 presentes en los sedimentos portadores, permitieron obtener una datación radiocarbónica de $8.480 \pm 130$ años ${ }^{14} \mathrm{C} \mathrm{AP}$ (LP2399). La fractura, que separó la epífisis RPCR 1021 del resto del húmero (no recuperado), muestra bordes angulosos. Esto, sumado a que la epífisis no presenta marcas de transporte y a que las fracturas en el tejido esponjoso expuesto también son angulosas, permite proponer que la fractura puede haber sido originada muy recientemente; con toda probabilidad, producida por la máquina utilizada para la remoción del sedimento en la cantera, ya que sus paredes presentan marcas dejadas por las uñas de dicha máquina.

\section{ÁREA DE ESTUDIO Y GEOLOGÍA}

El ejemplar estudiado proviene de la cantera "Nicolás Vignogna III" ubicada en Los Pozos, partido de Marcos Paz, Provincia de Buenos Aires (Figura 1). El nivel portador (nivel A, Figura 2) contiene abundantes valvas de Biomphalaria sp., presenta estructura laminar que sugiere depósitos de albardón en planicie de inundación y constituye el material parental de un suelo incipiente. El ejemplar aquí estudiado estaba claramente asociado a las valvas de Biomphalaria sp. al momento de su hallazgo. Por debajo (nivel B, Figura 2), se observa un cuerpo laminar con rasgos ambientales análogos al nivel portador; se lo interpreta como un depósito de planicie de inundación. Infrayaciendo a este nivel y en discordancia se observan unas arenas consolidadas, masivas (nivel C, Figura 2).

\section{MATERIAL Y MÉTODOS}

Las valvas de Biomphalaria sp. fueron datadas en el Laboratorio de Tritio y Radiocarbono (LATYR; Centro de Investigaciones Geológicas, Facultad de Ciencias Naturales y Museo, UNLP y CONICET).

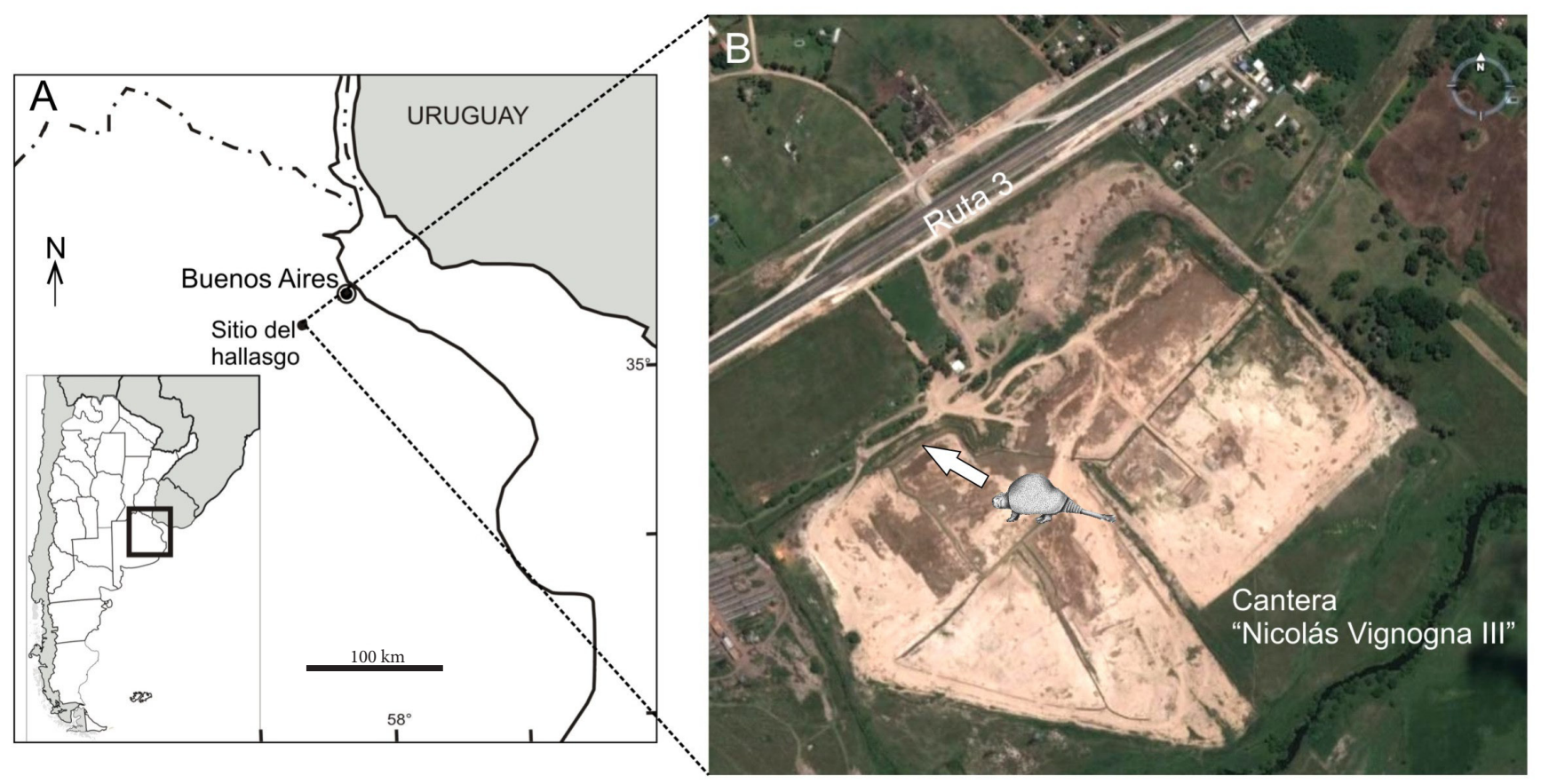

Figura 1. A, mapa de ubicación del sitio de hallazgo; B, foto aérea de la cantera "Nicolás Vignogna III", la flecha indica el punto de coleta del material.

Figure 1. A, location map of the outcrop; B, aerial photo of the quarry "Nicolás Vignogna III", the arrow indicates the point from where material was colected. 


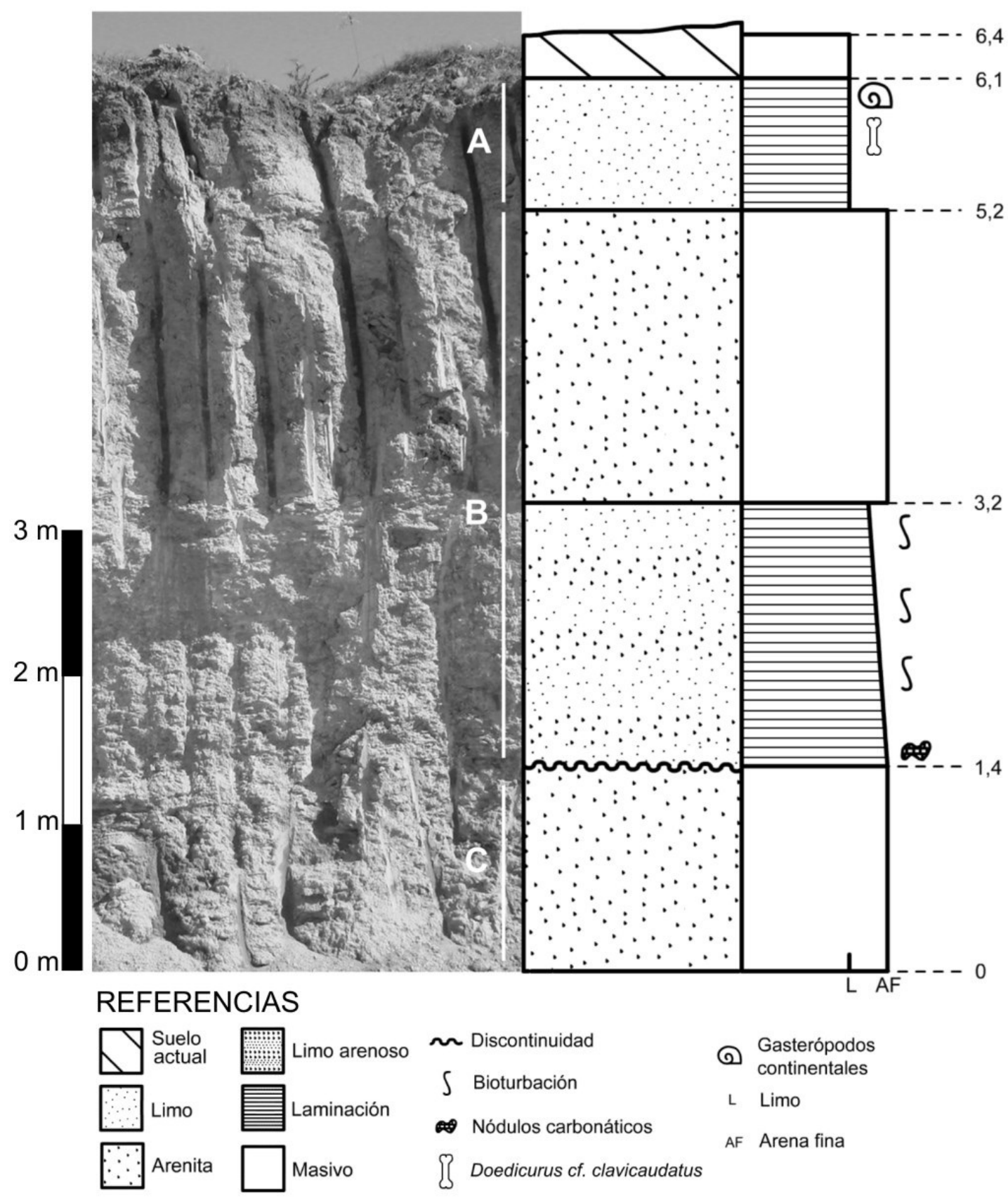

Figura 2. Perfil estratigráfico del sector estudiado. A, nivel donde el ejemplar RPCR 1021 estaba claramente asociado a las valvas de Biomphalaria sp.; B, en este nivel se observa un cuerpo laminar con rasgos ambientales análogos al nivel A; C, este nivel se encuentra en discordancia con el nivel $B$.

Figure 2. Stratigraphic profile of the studied sector. A, level where the specimen RPCR 1021 was clearly associated with the valves of Biomphalaria sp.; B, this level shows a body laminar with analogous environmental features to level A; C; this level is in discordance with the level B.

En el material estudiado (RPCR 1021) no se pueden tomar, en forma directa, las medidas que en los húmeros de gliptodontes, y de otros de mamíferos, se utilizan para calcular la masa corporal (ver Fariña et al., 1998; Fariña, 2000; Elissamburu, 2004; Elissamburu \& Vizcaíno, 2004, 2005). Sin embargo, es posible inferir la masa corporal de un ejemplar asumiendo la similitud geométrica existente entre dos taxones muy emparentados. La ecuación utilizada indica la masa potencial de un individuo problema (en este caso RPCR 1021) por comparación geométrica con un individuo de morfología semejante y masa conocida (en este caso Doedicurus clavicaudatus) y ha sido recientemente utilizada para estimar la masa de diferentes taxones (e.g. Vizcaíno et al., 2006; Soibelzon \& Tartarini, 2009):

$$
\mathrm{M}_{\mathrm{X}} / \mathrm{M}_{\mathrm{Dc}}=\left(\mathrm{Lx}_{\mathrm{X}} / \mathrm{Lx}_{\mathrm{Dc}}\right)^{3}
$$

De donde $\mathrm{M}_{\mathrm{X}}=\left(\mathrm{Lx}_{\mathrm{X}} / \mathrm{Lx}_{\mathrm{Dc}}\right)^{3 *} \mathrm{M}_{\mathrm{Dc}}$ $\mathrm{M}_{\mathrm{X}}=$ masa del ejemplar problema.

$\mathrm{M}_{\mathrm{Dc}}=$ masa de Doedicurus clavicaudatus .

$\mathrm{Lx}_{\mathrm{X}}=$ medida $\mathrm{x}$ en el ejemplar problema.

$\mathrm{Lx}_{\mathrm{Dc}}=$ medida $\mathrm{x}$ en $D$. clavicaudatus.

En la fórmula se reemplazaron los términos por las 
medidas relevadas sobre el ejemplar MLP 16-24 (Doedicurus clavicaudatus) y las extraídas del ejemplar RPCR 1021 (Figura 3A). La masa corporal de D. clavicaudatus (1400 $\mathrm{kg}$ ) fue tomada de Fariña et al. (1998).

Para realizar la descripción del material y la comparación de éste con regiones homólogas pertenecientes a especies afines se siguió la terminología anatómica utilizada por Sisson \& Grossman (1959). Asimismo, para características peculiares de los gliptodontes, se consultó Burmeister (1874), Gillete \& Ray (1981) y Zurita (2007). Se utiliza “cf." en el sentido dado por Bengtson (1988).

Materiales utilizados en las comparaciones: Doedicurus: MLP 42-VI-24-10, MLP 55-V-3-2, MLP 55-VIII-12-18, MLP 04-X-3-1; MLP 16-24 (D. clavicaudatus); Panochthus Burmeister, 1866: MLP 16-29, MLP 16-38; MLP 16-115; MLP 16-216; MLP 10-VIII-4-1 (P. tuberculatus Owen, 1845); Glyptodon: MLP 07-IX-21, MLP 16-144; MLP 16-41; MLP 16-44 (G. clavipes), MLP 10-VIII-5-1 (G. reticulatus Owen, 1845).

Abreviaturas institucionales. MLP, Colección de la División Paleontología de Vertebrados del Museo de La Plata, Buenos Aires, Argentina; RPCR, Repositorio Paleontológico "Carlos Rusconi” del Museo Histórico Municipal de La Matanza "Brigadier General Don Juan Manuel de Rosas"; Buenos Aires, Argentina.

\section{PALEONTOLOGÍA SISTEMÁTICA}

Superorden XENARTHRA Cope, 1889

Orden CINGULATA Illiger, 1811

Superfamilia GLYPTODONTOIDEA Burmeister, 1879

Familia GLYPTODONTIDAE Gray, 1869

Subfamilia DOEDICURINAE Ameghino, 1889

Doedicurus Burmeister, 1874

\section{Doedicurus cf. D. clavicaudatus (Owen, 1847)}

(Figuras 3A-F)

Material referido. Epífisis distal del húmero derecho RPCR 1021 (Figura 3) proveniente del perfil noroeste de la Cantera "Nicolás Vignogna III" (Figura 1A-B), Marcos Paz, Buenos

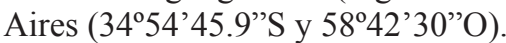

Procedencia estratigráfica y antigüedad. Nivel A (Figura 2), $8.480 \pm 130$ años ${ }^{14} \mathrm{C} \mathrm{AP}(9.590-9.327$ años cal. AP), Holoceno temprano.
Descripción y comparaciones. Se conservan los cóndilos medial y lateral completos (cóndilo interno y lateral respectivamente de Zurita, 2007). El epicóndilo medial está roto, pero se puede inferir en qué dirección se proyectaría. El epicóndilo lateral se conserva en su totalidad. El resto preservado mide $110 \mathrm{~mm}$ longitudinalmente y $137 \mathrm{~mm}$ transversalmente. En vista anterior (Figura 3B), por sobre el borde condilar, entre el medial y el lateral, se aprecia una muy leve depresión que corresponde a la fosa coronoides. Desde esta vista, a $20 \mathrm{~mm}$ del borde del cóndilo medial y a $53 \mathrm{~mm}$ del cóndilo lateral, la superficie del hueso está rota. La fosa coronoides es similar a la de Doedicurus clavicaudatus y Panochthus tuberculatus; en Glyptodon reticulatus, dicha fosa está mucho más excavada. En vista posterior (Figura 3C), por encima del borde condilar, entre el medial y el lateral, aparece la fosa olecraneana, ésta es de contorno subelíptico y tan profunda como en $D$. clavicaudatus y P. tuberculatus. En cambio, la fosa olecraneana de G. reticulatus es mucho más profunda y de contorno subtriangular. Desde esta vista se puede apreciar mayor superficie de hueso intacto, pues está roto a $64 \mathrm{~mm}$ del borde del cóndilo medial y a 43 mm del borde del lateral (Figuras 3E,F). Estas últimas medidas calculadas son aproximadas porque los bordes respectivos son irregulares (Figura 3A).

En la Figura 3A se observa una representación gráfica de las medidas que se consideraron más significativas. En la Tabla 1 se muestran los resultados de las medidas del ejemplar RPCR 1021 y aquéllas de las regiones homólogas en ejemplares de Glyptodon reticulatus, Panochthus tuberculatus y Doedicurus clavicaudatus. En vista distal (Figura 3D), puede observarse que las dimensiones de esta superficie son mayores en el material RPCR 1021 que en los restos de las otras especies aquí comparadas (Tabla1). El borde del cóndilo medio sobresale interiormente más que en $P$. tuberculatus y $G$. reticulatus, a semejanza de $D$. clavicaudatus. El diámetro mínimo antero-posterior (Figura 3A) coincide con la constricción intercondílea; ésta es de igual aspecto que la de $D$. clavicaudatus y similar a la de $P$. tuberculatus, en tanto que en $G$. reticulatus dicha constricción resulta mucho más acentuada. La masa corporal estimada para el ejemplar RPCR 1021 se encuentra en el rango 1900 a 2370 kg según cuál sea la medida utilizada (Figura 3A, Tabla 1) mientras que la estimada para D. clavicaudatus es de 1400 $\mathrm{kg}$ (ver Fariña et al., 1998). De lo expuesto se desprende que el ejemplar RPCR 1021 sólo se diferencia de los ejemplares estudiados de D. clavicaudatus por su tamaño mayor.

Tabla 1. Cuadro comparativo de medidas del ejemplar RPCR 1021 y ejemplares de otras especies. Adicionalmente se brinda la masa del ejemplar RPCR 1021 estimada por similitud geométrica a partir de cada medida considerada (ver Material y Métodos). Medidas lineales en mm. Abreviaturas: DMAP, diámetro mínimo antero-posterior; DMCL, diámetro máximo del cóndilo lateral; DMCM, diámetro máximo del cóndilo medial; DTC, diámetro transverso del cóndilo.

Table 1. Comparative measures of the RPCR 1021 specimen and specimens of other species. In addition gives the mass of the RPCR 1021 estimated by geometric similarity from each measure considered (see Material and Methods). Linear measures in mm. Abbreviations: DMAP, minimum anteroposterior diameter; DMCL, maximum diameter of the lateral condyle; DMCM, maximum diameter of the medial condyle; DTC transverse diameter of the condyle.

\begin{tabular}{|c|c|c|c|c|}
\hline Taxa $\$ Medidas & DMAP & DMCL & DMCM & DTC \\
\hline Doedicurus clavicaudatus (MLP 16-24) & 46 & 47 & 56 & 77 \\
\hline Panochthus tuberculatus (MLP 10-VIII-4-1) & 40 & 43 & 51 & 72 \\
\hline Glyptodon reticulatus (MLP 10-VIII-5-1) & 40 & 46 & 57 & 81 \\
\hline \multirow{2}{*}{$\begin{array}{l}\text { medida } \\
\text { masa estimada }(\mathrm{kg})\end{array}$} & 51 & 56 & 66 & 86 \\
\hline & 1908 & 2368 & 2292 & 1951 \\
\hline
\end{tabular}



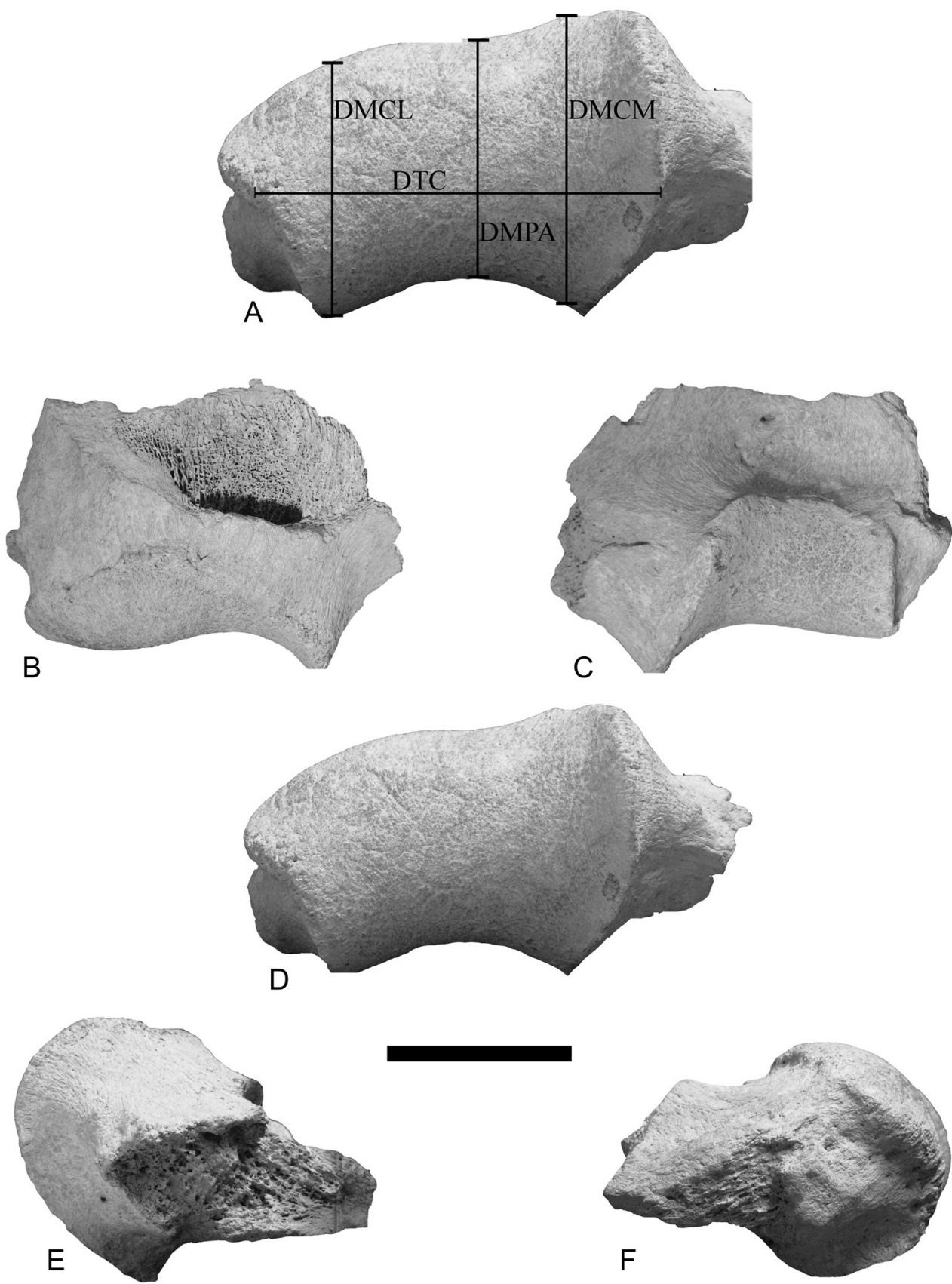

Figura 3. Epífisis distal del húmero derecho de Doedicurus cf. C. clavicaudatus (RPCR 1021). A, medidas relevadas en la superficie distal; B, vista anterior; C, vista posterior; D, vista de la superficie distal; E, vista medial; F, vista lateral. Abreviaturas: DMAP, diámetro mínimo antero-posterior; DMCL, diámetro máximo del cóndilo lateral; DMCM, diámetro máximo del cóndilo medial; DTC, diámetro transverso del cóndilo. Escala $=50 \mathrm{~mm}$.

Figure 3. Distal epiphysis of right humerus of Doedicurus cf. C. clavicaudatus (RPCR 1021). A, measures taken in the distal surface; B, anterior view; C, posterior view; D, distal surface; E, medial view; F, lateral view. Abbreviations: DMAP, minimum anteroposterior diameter; DMCL, maximum diameter of the lateral condyle; DMCM, maximum diameter of the medial condyle; DTC, transverse diameter of the condyle. Scale bar $=50 \mathrm{~mm}$.

\section{DISCUSIÓN}

Scillato-Yané et al. (1995), Carlini \& Scillato-Yané (1999) y Cione et al. (1999) mencionan para el Lujanense (Pleistoceno tardío a Holoceno temprano) de la Argentina, tres géneros de gliptodontes con especies cuya masa es igual o mayor a los 1000 kg. Estos son Panochthus, Glyptodon y Doedicurus. A esta lista de taxones con masas iguales o superiores a 1 tonelada, se le suma Plaxhaplous Ameghino, 1884 (Vizcaíno et al., en prensa).

Las especies de todos estos géneros fueron habitantes de áreas abiertas de pastizales, con climas templados y templados fríos (Scillato-Yané et al., 1995; Zurita et al., 2009). 
Algunas estimaciones cuantitativas de la masa corporal de los gliptodontes han conducido a resultados sólo parcialmente coincidentes con los citados. Así, Fariña (2000) considera que los gliptodontes de mayor masa corporal son Doedicurus clavicaudatus y Glyptodon clavipes. En cambio, Pasquali \& Tonni (2004, p. 38) afirman que "La coraza de $G$. clavipes es relativamente pequeña comparada con las de otros gliptodontes (...)". Una explicación posible a estas discrepancias radica en el hecho que las colecciones paleontológicas originalmente alojadas en el College of Surgeons (Londres), sufrieron deterioros durante la Segunda Guerra Mundial. Fariña (2000, p. 51) estimó en 2000 kg la masa de G. clavipes, pero, posteriormente ese autor duda de la identificación específica del material estudiado (R. Fariña, com. pers. a G.J.S.-Y.). Esta duda se basa en que en las antiguas colecciones del College of Surgeons, se encontraba una coraza de G. elongatus, que pudo haber sido atribuida a $G$. clavipes y sobre esa base construido el modelo en el que se basó Fariña (2000) para su estimación de masa. En suma, es probable que el ejemplar cuyo peso fue estimado por Fariña (2000) en $2000 \mathrm{~kg}$ pertenezca a G. elongatus y no a $G$. clavipes.

De esta manera, el ejemplar Doedicurus cf. D. clavicaudatus RPCR 1021 estaría, junto con Panochthus intermedius, P. subintermedius Castellanos, 1937, Glyptodon munizi Ameghino, 1881, Plaxhaplous canaliculatus (Ameghino, 1884), G. elongatus y Doedicurus clavicaudatus, entre los mayores gliptodontes conocidos (Scillato-Yané et al., 1995; Scillato-Yané \& Carlini, 1998; Cione \& Tonni, 2005; Soibelzon et al., 2006, Cruz et al., 2010; Vizcaíno et al., en prensa).

Las tres primeras especies mencionadas son exclusivas del Pleistoceno temprano, mientras que las otras se registran durante el Pleistoceno tardío y Holoceno temprano. Esto sugiere que durante el Cuaternario se verificaron dos momentos en que los Glyptodontidae contaron con formas de gran tamaño. Panochthus con sus especies de mayor masa en el Pleistoceno temprano, constituye la única excepción.

Soibelzon et al. (2009) y luego Zurita et al. (2010) sugirieron que el arribo a América del Sur de los grandes predadores holárticos (e.g. osos de rostro corto y tigres diente de sable) a comienzos de Pleistoceno, en el marco del Gran Intercambio Biótico Americano (GIBA) debió haber desatado una serie de respuestas adaptativas entre los herbívoros autóctonos como el aumento del tamaño corporal y el desarrollo de estructuras accesorias de defensa como respuesta a la presión de predación.

Se descarta que el gran tamaño alcanzado por los gliptodontes del Pleistoceno tardío-Holoceno temprano sea explicable por la "Regla de Bergman" ya que el Holoceno comienza en 11.700 años cal. AP, es decir cuando finaliza la Reversión Fría Antántica-Younger Dryas; a partir de ese momento se registra un incremento global en las temperaturas que genera en torno a 10.000 años cal. AP una ingresión marina. La tendencia se revierte parcialmente hace 8.000 años cal. AP con un enfriamiento que rápidamente (en torno a 7.500 años cal. AP) da lugar al máximo térmico del Holoceno que dura hasta el Neoglacial (es decir hasta $c a$.
4.000 años cal. AP). En tanto, Vizcaíno et al. (en prensa) sostienen que el incremento en tamaño corporal pudo haber favorecido a estos taxones en la competencia con los linajes de placentarios pastadores holárticos (ingresados durante el GIBA). Según Vizcaíno et al. (2006) el bajo metabolismo les habría permitido a los xenartros alcanzar grandes tamaños con una incorporación mucho menor de alimento. Alternativamente, el desarrollo de grandes masas corporales pudo ser adaptativamente significativo para escapar de la predación por parte del hombre (presente desde al menos 14.220 y 13.980 años cal. AP; Dillehay et al., 2008) y de los grandes carnívoros de abolengo holártico, que se registran desde el Pleistoceno temprano (ver arriba).

\section{AGRADECIMIENTOS}

A.A. Carlini, S.F. Vizcaíno y A. Zurita por el asesoramiento brindado en distintas cuestiones; M. Reguero y L. Pomi, por facilitarnos el estudio de los ejemplares depositados en las colecciones del MLP. A la Secretaría de Cultura de la municipalidad de La Matanza por colaborar con la logística de las campañas. A los dos revisores anónimos y A.M. Ribeiro por sus valiosos comentarios. Esta es una contribución a los proyectos PICT 2010/1664, PICT 0804, PROSUL-CNPq 490299/2008-3 y PIP 0436.

\section{REFERENCIAS}

Ameghino, F. 1881. La formación pampeana o studio sobre los terrenos de transporte de la cuenca del Plata. G. Masson-Igon Hermanos, Paris y Buenos Aires, 376 p.

Ameghino, F. 1884. Excursiones geológicas y paleontológicas en la Provincia de Buenos Aires. Boletín de la Academia Nacional de Ciencias en Córdoba, 6:161-257.

Bengtson, P. 1988. Open nomenclature. Palaeontology, 31:223-227.

Burmeister, G. 1866. Lista de los mamíferos fósiles del terreno diluviano. Anales del Museo Público de Buenos Aires, 1:121-232.

Burmeister, G. 1874. Monografía de los glyptodontes en el Museo Público de Buenos Aires. Anales del Museo Público de Buenos Aires, 2:355-412.

Carlini, A.A. \& Scillato-Yané, G.J. 1999. Evolution of Quaternary Xenarthrans (Mammalia) of Argentina. Quaternary of South America and Antarctic Peninsula, 12:131-156.

Carlini, A.A. \& Zurita, A.E. 2010. An introduction to Cingulate evolution and their evolutionary history during the Great American Biotic Interchange: biogeographical clues from Venezuela. Urumaco and Venezuela Paleontology. In: M. Sanchez-Villagra; O. Aguilera \& A.A. Carlini (eds.) The Fossil Reccord of the Northern Neotropics, Indiana University Press, p. 233-255.

Cione, A.L. \& Tonni, E.P. 2005. Bioestratigrafía basada en mamíferos del Cenozoico superior de la provincia de Buenos Aires, Argentina. CONGRESO GEOLÓGICO ARGENTINO, 16, 2005. Relatorio, La Plata, 11, p. 183-200.

Cione, A.L.; Tonni, E.P.; Bond, M.; Carlini, A.; Pardiñas, U. F.J.; Scillato Yané, G.; Verzi, D. \& Vucetich, M.G. 1999. Occurrence charts of Pleistocene mammals in the Pampean area, eastern Argentina. In: E.P. Tonni \& A.L. Cione (eds.) Quaternary of South America and Antarctic Peninsula, Balkema, 12:53-73.

Cione, A.L.; Tonni, E.P. \& Figini, A.J. 2001. Did the megafauna range to 4,300 yr BP in South America? Radiocarbon, 43:69-75. 
Cione, A.L.; Tonni, E.P. \& Soibelzon, L.H. 2009. Did humans cause the late Pleistocene-early Holocene mammalian extinctions in South America in a context of shrinking open areas? In: G. Haynes (ed.) American megafaunal extinctions at the end of the Pleistocene, Vertebrate Paleobiology and Paleoanthropology Series, Springer, p. 125-144.

Coltorti, M.; Della Fazia, J.; Paredes Ríos, F. \& Tito, G. 2012. Ñuagapua (Chaco, Bolivia): Evidence for the latest occurrence of megafauna in association with human remains in South America. Journal of South American Earth Sciences, 33:56-67. doi:10.1016/j.jsames.2011.07.003

Cruz, L.E.; Zamorano, M. \& Scillato-Yané, G.J. 2010. Diagnosis and redescription of Panochthus subintermedius Castellanos (Xenarthra, Glyptodontia) from the Ensenadan (early-middle Pleistocene) of Buenos Aires (Argentina). Neues Jahrbuch für Geologie und Palaeontologie Abhandlungen, 85:115-123. doi: 10.1007/s12542-010-0084-7

Dillehay, T.D.; Ramírez, C.; Pino, M.; Collins, M.B.; Rossen, J. \& Pino-Navarro, J.D. 2008. Monte Verde: Seaweed, Food, Medicine, and the Peopling of South America. Science, 320:784-786. doi:10.1126/science. 1156533

Elissamburu, A. 2004. Análisis morfométrico y morfofuncional del esqueleto apendicular de Paedotherium (Mammalia, Nototungulata). Ameghiniana, 41:363-380.

Elissamburu, A. \& Vizcaíno, S.F. 2004. Limb proportions and adaptations in caviomorph rodents (Rodentia: Caviomorpha). Journal of Zoology, 262:145-159.

Elissamburu, A. \& Vizcaíno, S.F. 2005. Diferenciación morfométrica del húmero y fémur de las especies de Paedotherium (Mammalia, Notoungulata) del Plioceno y Pleistoceno temprano. Ameghiniana, 42:159-167.

Fariña, R.A. 1995. Limb bone strength and habits in large glyptodonts. Lethaia, 28:189-196.

Fariña, R.A. 2000. Física y matemáticas para reconstruir la vida en el pasado. Actas de Fisiología, 6:45-70.

Fariña, R.A.; Vizcaíno S.F. \& Bargo, M.S. 1998. Body mass estimations in Lujanian (late Pleistocene-early Holocene of South America) mammal megafauna. Mastozoología Neotropical, 5:87-108.

Fariña, R.A. \& Vizcaíno, S.F. 1999. A century after Ameghino: the palaeobiology of the large Quaternary mammals of South America revisited. In: E.P. Tonni \& A.L. Cione (eds.) Quaternary of South America and Antarctic Peninsula, Balkema, 12:255-277.

Gilette, D.D. \& Ray, C.E. 1981. Glyptodonts of North America. Smithsonian Contributions to Palaeobiology, 40:1-251.

Gutierrez, M.A.; Martyinez, G.A.; Bargo, M.S. \& Vizcaíno, S. 2010. Supervivencia diferencial de mamíferos de gran tamaño en la región pampeana en el Holoceno temprano y su relación con aspectos paleobiológicos. In: M.A. Gutierrez; M. De Nigris; P.M. Fernández; M. Giardina; A. Gil; A. Izeta; G. Neme \& H. Yacobaccio (eds.) Zooarqueología a principios del siglo XXI: aportes teóricos, metodológicos y casos de estudio, Ediciones del Espinillo, p. 231-241.

Owen, R. 1839. Note on the Glyptodon. In: W. Parish (ed.) Buenos Aires and the Provinces of the Rio de La Plata, p. 1-178.

Owen, R. 1845. Descriptive and illustrated catalogue of the fossil organic remains of Mammalia and Aves contained in the Museum of the Royal College of Surgeons of London. London, R. \& J.E. Taylor, $391 \mathrm{p}$.

Owen, R. 1847. Notices of some fossil Mammalia of South America. In: MEETING OF THE BRITISH ASSOCIATION FOR THE ADVANCEMENT OF SCIENCE, 16, 1846. Report, Southampton, p. 65-67.
Pasquali, R.C. \& Tonni, E.P. 2004. Los mamíferos fósiles de Buenos Aires, cuando los gliptodontes caminaban por la Avenida de Mayo. Córdoba, Universitas, 146 p.

Paula Couto, C. 1957. Sôbre um gliptodonte do Brasil. Boletim Divisão de Geología e Mineralogía, 165:1-37.

Paula Couto, C. 1979. Tratado de Paleomastozoologia. Rio de Janeiro, Academia Brasileira de Ciencias, 590 p.

Politis, G. \& Gutiérrez, M. 1998. Gliptodontes y cazadores-recolectores de la Región Pampeana (Argentina). Latin American Antiquity, 9:111-134.

Rosello, E.A; Jahn, B-M; Liu, T. \& Petrocelli, J.L. 1999. New $4,300 \mathrm{YR}^{14} \mathrm{C}$ age of glyptodonts at Luján River (Buenos Aires, Argentina) and its implications. In: SOUTH AMERICAN SYMPOSIUM ON ISOTOPE GEOLOGY, 2, 1999. Actas, Buenos Aires, p. 105-110.

Scillato-Yané, G.J. \& Carlini, A.A. 1998. Un gigantesco Gliptodonte en los alrededores de la Ciudad de La Plata. Facultad de Ciencias Naturales y Museo de La Plata, 11:45-48.

Scillato-Yané, G.J.; Carlini, A.A.; Vizcaíno, S.F. \& Ortiz-Jaureguizar, E. 1995. Xenarthra. In: M.T. Alberdi; E.P. Tonni \& G. Leone (eds.) Evolución biológica y climática de la región pampeana durante los últimos cinco millones de años. Un ensayo de correlación con el Mediterraneo Occidental, Museu Nacional de Ciencias Naturales, Consejo Superior de Investigaciones Científicas, Monografías, 9:183-211.

Sisson, S. \& Grossman, J.D. 1959. Anatomía de los animales domésticos. 5ta. ed. Barcelona, Salvat Editores, 2302 p.

Soibelzon, E.; Zurita, A.E. \& Carlini, A.A. 2006. Glyptodon munizi (Mammalia, Cingulata, Glyptodontidae): redescripción y anatomía. Ameghiniana, 43:377-385.

Soibelzon, L.H.; Pomi, L.M.; Tonni, E.P.; Rodriguez, S. \& Dondas, A. 2009. First report of a short-faced bears' den (Arctotherium angustidens). Palaeobiological and Palaeoecological implications. Alcheringa, 33:211-222. doi: 10.1080/03115510902844418

Soibelzon, L.H. \& Tartarini, V.B. 2009. Estimación de la masa corporal de las especies de osos fósiles y actuales (Ursidae, Tremarctinae) de América del Sur. Revista Museo Argentino de Ciencias Naturales "Bernardino Rivadavia”, 11:243-254.

Vizcaíno, S.F. \& Bargo, M.S. 1998. The masticatory apparatus of Eutatus (Mammalia, Cingulata) and some allied genera. Evolution and paleobiology. Paleobiology, 24:371-383.

Vizcaíno, S.F.; Bargo, M.S. \& Cassini, G.H. 2006. Dental occlusal surface area in relation to body mass, food habits and other biological features in fossil xenarthrans. Ameghiniana, 43:11-27.

Vizcaíno, S.F.; Cassini, G.H.; Toledo, N. \& Bargo, M.S. En prensa. On the evolution of large size in mammalian herbivores of Cenozoic faunas of Southern South America. In: B.D. Patterson \& L.P. Costa (eds.) Bones, clones, and biomes: an 80-million year history of Recent neotropical mammals, University of Chicago Press, p. 76-101.

Vizcaíno, S.F.; Fariña, R.A.; Bargo, M.S. \& De Iuliis, G. 2004. Functional and phylogenetical assessment of the masticatory adaptations in Cingulata (Mammalia, Xenarthra). Ameghiniana, 41:651-664.

Zurita,A.E. 2007. Sistemática yevolución de los Hoplophorini (Xenarthra, Glyptodontidae, Hoplophorinae. Mioceno tardio-Holoceno temprano). Importancia bioestratigráfica, paleobiogeográfica y paleoambiental. Programa de Doctorado en Ciencias Naturales, Universidad Nacional de La Plata, Tesis Doctoral, 367 p.

Zurita A.E.; Miño-Boilini, A.R.; Soibelzon, E.; Carlini, A.A. \& Paredes Ríos, F. 2009. The diversity of Glyptodontidae (Xenarthra, Cingulata) in the Tarija Valley (Bolivia): systematic, biostratigraphic 
and paleobiogeographic aspects of a particular assemblage. Neues Jahrbuch für Geologie und Paläontologie, 251/252:225-237. doi: 10.1127/0077-7749/2009/0251-0225

Zurita, A.E.; Scarano, A.C.; Carlini, A.A.; Scillato-Yané, G.J. \& Soibelzon,

E. 2011. Neosclerocalyptus spp. (Cingulata, Glyptodontidae,
Hoplophorini): cranial morphology and palaeoenvironments, along the changing Quaternary. Journal of Natural History, 45:893-914. doi: 10.1080/00222933.2010.536917

Received in June, 2011; accepted in February, 2012. 